\title{
Familial Hyperlipidemia Screening, Treatment and New Considerations
}

\author{
Shauna Wentzell ${ }^{\mathrm{a}, \mathrm{d}}$, Mary Ryan ${ }^{\mathrm{a}, \mathrm{b}}$, Vivion Crowley ${ }^{\mathrm{c}}$
}

\begin{abstract}
We present a case of a 47-year-old male who came to the endocrinology department at the request of his GP. He was noted to have elevated lipid levels, twice the normal range. Genetic testing was performed which showed he was heterozygous for familial hypercholesterolemia (FH). His three children subsequently had their lipid levels tested. They all had elevated lipids (cholesterol and LDL) and tested positive for FH. This case highlights the importance of lipid screening in children as the prevalence of FH is 1 in 200, which is higher than previously thought. This has led to the underdiagnosis of $\mathrm{FH}$ in both the pediatric and adult population. Often on initial presentation of the disease, irreversible damage has been done increasing the patients' morbidity and mortality. With newer drugs being introduced and the persistent push by the National Heart, Lung and Blood Institute Expert Panel towards initiating a child screening protocol, there is hope that this disease will be manageable and less detrimental to those affected.
\end{abstract}

Keywords: Familial hyperlipidemia; PCSK9 inhibitor; Genetic screening; Lipid

\section{Introduction}

This case was written to highlight the increasing prevalence of familial hyperlipidemia and the genetic relationship between families. It is meant to educate readers of the potential benefits of early childhood screening in reducing the morbidity and mortality of this condition. Early treatment implementation is crucial for those affected and advancements in drug therapies, specifically PCSK9 inhibitors, have shown promising results.

\section{Case Report}

A 47-year-old male of Irish heritage presented to the endocri-

Manuscript accepted for publication May 11, 2016

aUniversity of Limerick Graduate Entry Medical School, Ireland

bBarrington's Hospital Limerick, Ireland

'Department of Biochemistry, St. James's Hospital, Dublin, Ireland

${ }^{\mathrm{d} C}$ Corresponding Author: Shauna Wentzell, University of Limerick Graduate

Entry Medical School, Ireland. Email: Wentzell.shauna@gmail.com

doi: http://dx.doi.org/10.14740/jmc2533w nology department on September 5. 2015 with concerns of a raised blood total cholesterol of $10.8 \mathrm{mmol} / \mathrm{L}$. With a cholesterol level being double the normal limit $(5.2 \mathrm{mmol} / \mathrm{L})$, there was concern of some genetic involvement.

Genetic testing was performed on September 11, 2015, indicating the patient was heterozygous for a mutation p.E57K in the LDLR gene. This variant has been reported as causing a genetic susceptibility to familial hypercholesterolemia (FH). As this is an autosomal dominant condition, he was encouraged to have his offspring tested for the genetic mutation.

Genetic testing was performed on his three biological children aged 11, 12 and 13 years. Results indicated all three of his children had inherited the p.E57K mutation in keeping with the autosomal dominant inheritance pattern of the disease. Blood lipid levels were obtained from each child which further supported this diagnosis as all three children had significantly elevated serum total cholesterol $(5.6 \mathrm{mmol} / \mathrm{L}, 5.1 \mathrm{mmol} / \mathrm{L}$, and $5.3 \mathrm{mmol} / \mathrm{L})$ and LDL cholesterol levels $(5.3 \mathrm{mmol} / \mathrm{L}, 7.6$ $\mathrm{mmol} / \mathrm{L}$, and $6.05 \mathrm{mmol} / \mathrm{L}$ ).

Given the importance of obtaining a correct diagnosis, a repeat genetic test was performed to ensure accurate results. As expected, this reconfirmed the previous result.

Due to the consequences of uncontrolled lipid levels, all patients were placed on statins. The father was also placed on ezetimibe to further improve his elevated cholesterol levels. A new family of drugs called PCSK9 inhibitors will be launched in Ireland in 2016. These PCSK9 inhibitors are showing tremendous results for reducing lipid levels in FH patients [1]. Should his current treatment not adequately lower his lipid levels, it is very likely the father may be offered a PCSK9 inhibitor once it becomes available.

\section{Discussion}

FH is a genetic disorder which has an autosomal dominant inheritance pattern and is more common than previously thought. Work done by De Ferranti [2] estimated that the prevalence of FH was roughly 1 in 500 persons; however, more recent evidence suggests it is more accurately 1 in 200 persons [3].

The most common form of FH is the heterozygous form. This is often asymptomatic until adulthood. Most patients develop early cardiac events as there is a 20 -fold increase in atherosclerosis cardiovascular disease mortality [2]. In very rare cases, FH can present in a homozygous form. These patients will present in early infancy with xanthelasmas and if not promptly diagnosed, can lead to myocardial infarction as early as 2 years of age [2].

As in this case report, children often remain undiagnosed 
until later in life when they present with major health events. It is for this reason that screening lipid levels in children has been recommended by the National Heart, Lung and Blood Institute Expert Panel [4]. With the prevalence of FH being higher than what was previously estimated, screening can provide youth with treatment options and hopefully this will lead to a reduced rate of premature cardiac events.

Treatment of FH is multifactorial. Lifestyle modification is the mainstay first line treatment in anyone with elevated cholesterol levels. A diet rich in fruit and vegetables yet low in saturated and trans fat is highly encouraged. Exercise is also beneficial and it is recommended that 30 min of daily physical activity for 5 days out of 7 has proven to be beneficial [2]. However, lifestyle modification can only go so far, especially where genetics are concerned. For most suffering from $\mathrm{FH}$, statins are the primary pharmacological treatment. In the pediatric $\mathrm{FH}$ population statins have shown to reduce LDL levels by $32 \%$ with some evidence of increasing HDL 3.4\% [5]. However, there is concern with the long-term safety of their use as there are limited data available on childhood statin therapy. Other agents commonly added to statin therapy are ezetimibe (as seen in this case report) and bile acid sequestrants. Their use has been shown to be beneficial for both the pediatric and adult population; however, adherence is a major concern due to side effects of bloating and constipation [2].

As mentioned in the above case, if the patient's lipid levels are not lowered sufficiently with his current treatment, our patient could be offered a PCSK9 inhibitor (protein convertase subtilisin kexin type 9) which binds to the LDL receptor promoting its degradation [1]. This is a novel treatment to be introduced this coming year in Ireland and appears to be an effective strategy to further lower the LDL cholesterol in patients with FH. Recent evidence published by the Canadian Prescriber's Newsletter [6] states that PCSK9 inhibitors can lower LDL levels of patients who are already receiving maximum statin dose. This does come at a significant financial cost to the patient as the drug is roughly $€ 5,000$ per annum and not likely to be universally covered by all drug plans. This class of drug is new and is currently being evaluated by the $\mathrm{Na}$ tional Institute for Health and Care Excellence (NICE). The programme director at NICE, Meindert Boysen, stated that although evolocumab (PCSK9 inhibitor) is effective in reducing levels of LDL cholesterol in people with hypercholesterolemia, it was felt that the drug does not have sufficient longterm data to support improvement of CVS outcomes nor does it have long-term safety data [7]. There are currently four longterm studies assessing the CVS outcomes; however, results of these studies are not expected until 2017 [8]. Hopefully these studies will help ascertain whether PCSK9 inhibitors will be a proven therapy option to decrease CVS morbidity and mortality in FH patients.

$\mathrm{FH}$ is notably a rising concern for our population. With its prevalence being higher than previously thought, it is sug- gested that lipid screening be instigated in childhood as early diagnosis is crucial to prevent the high rate of mortality and morbidity caused by the disease [9]. Evidence does suggest that early treatment is beneficial in reducing this CVS risk; however, more work needs to be done to bring this condition to the forefront. Often at the time of diagnosis, it is difficult to reverse the damage which has silently been wreaking havoc on the body for decades. With newer drugs being introduced and the persistent push towards initiating a child screening protocol, there is hope that this disease will become more manageable and less detrimental to those affected.

\section{References}

1. Hooper AJ, Burnett JR. Anti-PCSK9 therapies for the treatment of hypercholesterolemia. Expert Opin Biol Ther. 2013;13(3):429-435.

2. de Ferranti SD. Familial hypercholesterolemia in children and adolescents: A clinical perspective. J Clin Lipidol. 2015;9(5 Suppl):S11-19.

3. Nordestgaard BG, Chapman MJ, Humphries SE, Ginsberg HN, Masana L, Descamps OS, Wiklund O, et al. Familial hypercholesterolaemia is underdiagnosed and undertreated in the general population: guidance for clinicians to prevent coronary heart disease: consensus statement of the European Atherosclerosis Society. Eur Heart J. 2013;34(45):3478-3490a.

4. Integrated Guidelines for Cardiovascular Health and Risk Reduction in Children and Adolescents. National Heart, Lung and Blood Institute. Updated December 2011. Available at: www.nhlbi.nih.gov/guidelines/cvd_ped/index.htm. Accessed January 25, 2016.

5. Arambepola C, Farmer AJ, Perera R, Neil HA. Statin treatment for children and adolescents with heterozygous familial hypercholesterolaemia: a systematic review and meta-analysis. Atherosclerosis. 2007;195(2):339-347.

6. Murray A. New Drug: Repatha (Evolocumab). Canadian Prescriber's Letter. 2015;22(12):311230.

7. NICE issues draft guidance on evolocumab for lipid disorder. National Institute for Health and Care Excellence. Updated November 2015. Available at: https://www. nice.org.uk/news/press-and-media/nice-issues-draftguidance-on-evolocumab-for-lipid-disorder. Accessed on January 26, 2016.

8. Navarese EP, Kolodziejczak M, Schulze V, Gurbel PA, Tantry U, Lin Y, Brockmeyer M, et al. Effects of Proprotein Convertase Subtilisin/Kexin Type 9 Antibodies in Adults With Hypercholesterolemia: A Systematic Review and Meta-analysis. Ann Intern Med. 2015;163(1):40-51.

9. Nemati MH, Astaneh B. Optimal management of familial hypercholesterolemia: treatment and management strategies. Vasc Health Risk Manag. 2010;6:1079-1088. 